\title{
Simultaneous Lesion Segmentation and Bias Correction in Breast Ultrasound Images
}

\author{
Gerard Pons ${ }^{1}$, Joan Martí ${ }^{1}$, Robert Martí ${ }^{1}$, and J. Alison Noble ${ }^{2}$ \\ 1 Department of Computer Architecture and Technology, \\ University of Girona, Girona, Spain \\ \{gpons, joanm, marly\}@eia.udg.edu \\ 2 Department of Engineering Science, \\ University of Oxford, Oxford, United Kingdom \\ noble@robots.ox.ac.uk
}

\begin{abstract}
Ultrasound (US) B-mode images often show intensity inhomogeneities caused by an ultrasonic beam attenuation within the body. Due to this artifact, the conventional segmentation approaches based on intensity or intensity-statistics often do not obtain accurate results. In this paper, Markov Random Fields (MRF) and a maximum a posteriori (MAP) framework in combination with US image spatial information is used to estimate the distortion field in order to correct the image while segmenting regions of similar intensity inhomogeneity. The proposed approach has been evaluated using a set of 56 breast B-mode US images and compared to a radiologist segmentation.
\end{abstract}

Keywords: Breast lesion segmentation, ultrasound, inhomogeneity correction, Markov Random Fields, unsupervised initialization.

\section{Introduction}

Breast cancer is one of the leading causes of death for women all over the world and more than $8 \%$ women will suffer this disease during their life time 44. Detection in early stages of the cancer development is the key to reduce the death rate ( $40 \%$ or more) [3]. The earlier the cancers are detected, the better treatment can be provided. Currently, the most effective and used modality for breast cancer detection and diagnostic is digital mammography (DM) 3. However, there are some limitations of DM imaging in breast cancer detection, mostly in dense breasts where lesions can not be easily detected. Nowadays, an important alternative to DM is ultrasound (US) imaging, which is also commonly used as a complementary technique for breast cancer detection. One of the advantages of US against DM is its higher sensitivity when detecting lesions in dense breasts, reducing considerably the rate of false positives and thus the number of unnecessary biopsies. However, reading and understanding ultrasound images requires well-trained and experienced radiologists because of its complexity. Here lies the significance of the segmentation methods. An accurate and solid segmentation method is needed to, for instance, check if the lesion has grown through time. 
Several segmentation methods have been applied to breast US images, from the most traditional histogram thresholding methods [5|11] to novel approaches based on graph-cuts 7]12. Although active-contours methodologies are widely used 68 to determine the outline of the object of interest, they fail when dealing with blurred boundary lesions. Ultrasound image segmentation can also be considered as a labeling problem where the solution is to assign a set of labels to pixels, which is a natural representation for Markov Random Fields [2]10. Within this group we want to remark the work of Xiao et al. [10] who adapted a bias field removal method in magnetic resonance imaging (MRI) to segment abnormalities in breast B-mode US images. Precise and accurate results were obtained but the method required expert supervision to initialize the process. In this paper, we present different approaches to reduce the human interaction to one-click process and we evaluate these approaches and compare them to the original method using a data-set composed by breast US images with different lesion typologies.

\section{Methodology}

The method proposed by Xiao et al. considered the bias field as an additive artifact of the logarithmic ideal image. They estimated this field to restore the ideal image while at the same time identifying regions of similar intensity inhomogeneity using an MRF-MAP framework (see [10] for detail). Our proposal consists of combining this MRF-MAP framework with spatial information (see Fig. 1) in order to overcome the limitations of the method in terms of user interaction.

\subsection{Image Model}

The Xiao et al. proposal assumed that an attenuation-related intensity inhomogeneities is described as a multiplicative field with low-frequency. A logarithmic transformation of such multiplicative model yields an addition. Let $y$ and $y^{*}$ denote, respectively, the observed and ideal log-transformed intensities respectively, then $y=y^{*}+d$, where $d$ denotes the log-transformed intensity distortion field and $x_{i}$ denotes the corresponding class label of pixel $i$. A Gaussian distribution model is used to describe the intensity distribution in an image

$$
p\left(y_{i}^{*} \mid x_{i}\right)=g\left(y_{i}^{*} ; \theta\left(x_{i}\right)\right)
$$

where $g\left(y_{i}^{*} ; \theta\left(x_{i}\right)\right)$ is a Gaussian function and $\theta=\{\mu, \sigma\}$

Taking the distortion field into account and reformulating as a class-independent the intensity distribution (see Eq11) can be modeled as a Gaussian mixture

$$
p(y \mid d)=\prod_{i \in \mathcal{S}} \sum_{j \in \mathcal{L}} g\left(y_{i}-d_{i} ; \theta\left(x_{i}\right)\right) p\left(x_{i}=j\right)
$$

where $\mathcal{S}$ denotes the image pixels set and $d$ the distortion field. 


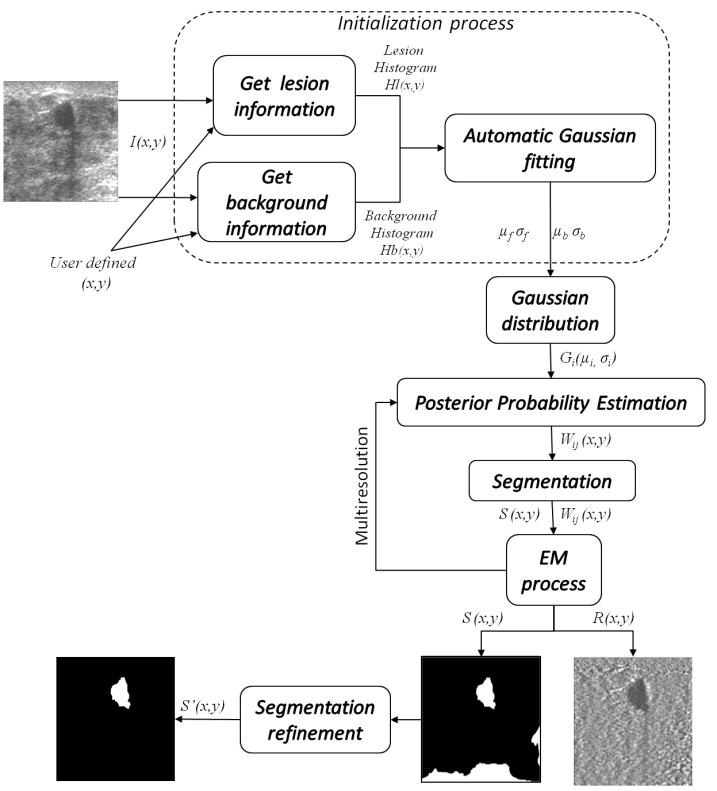

Fig. 1. Block diagram of our proposal, based on Xiao et al. [10] method

\subsection{Expectation-Maximization (EM) Algorithm}

As in Xiao et al. 10, Baye's rule can be used to obtain the posterior probability of the distortion field $(p(d \mid y))$, given the observed intensity values

$$
p(d \mid y)=\frac{p(y \mid d) p(d)}{p(y)}
$$

where $p(y)$ is a normalization factor and $p(d)$ is modeled as a Gaussian distribution with zero mean to capture its smoothness property. The maximum a posteriori principle is employed to obtain the optimal estimate of the distortion field. A zero-gradient condition is then used to assess this maximum, which leads to (see 9] for detail)

E step:

$$
W_{i j}=\frac{p\left(y_{i} \mid x_{i}, d_{i}\right) p\left(x_{i}=j\right)}{p\left(y_{i} \mid d_{i}\right)}
$$

M step:

$$
d_{i}=\frac{[F R]_{i}}{\left[F \psi^{-1} E\right]_{i}}, \text { with } E=(1,1, \ldots)^{T}
$$


where $W_{i j}$ is the posterior probability that pixel $i$ belongs to class $j$ given the distortion field estimate. $F$ is a low-pass filter and $R$ is the mean residual in which for pixel $i$

$$
R_{i}=\sum_{j \in \mathcal{L}} \frac{W_{i j}\left(y_{i}-\mu_{j}\right)}{\sigma_{j}^{2}}
$$

and $\psi$ is the mean inverse covariance

$$
\psi_{i k}^{-1}= \begin{cases}\sum_{j \in \mathcal{L}} W_{i j} \sigma_{j}^{-2} & \text { if } i=k \\ 0 & \text { if otherwise }\end{cases}
$$

As we said before, $W_{i j}$ is the posterior probability that pixel $i$ belongs to class $j$, and it is updated to the following form after using the MRF prior model

$$
W_{i j}=\frac{p\left(y_{i} \mid x_{i}, d_{i}\right) p\left(x_{i}=j \mid x_{\mathcal{N}_{i}}\right)}{p\left(y_{i} \mid d_{i}\right)}
$$

where $p\left(x_{i}=j \mid x_{\mathcal{N}_{i}}\right)$ has the form of Eq. 2

To obtain the estimation of the low-frequency distortion field, the EM algorithm is used to update one label image and intensity inhomogeneity field iteratively. Such an updating process converges rapidly in a few iterations. An ICM (Iterated Conditional Modes) algorithm [1] is used for this purpose.

\subsection{Automatic Initialization Process}

A major improvement on the original initialization process has been carried out. Different alternatives to obtain the Gaussian distribution parameters (mean and standard deviation) as much unsupervised as possible have been proposed, thus avoiding to empirically check the background and lesion histograms in order to manually fit the best Gaussian distribution to them. Actually, this lack of automatization in the original proposal of Xiao et al. prevented to properly evaluate that proposal since only one patient and one synthetic image were used, while the current proposal has been evaluated on several images from different patients. The initialization process starts by analyzing the intensity histogram of thee image and how the different Gaussian distributions fit to the lesion and background. Fig. 2 shows the histogram of a whole US image which almost fits perfectly to a Gaussian distribution. As far as we have experimented, always the histogram of the lesion is overlapped with the histogram of the background information. It is clear that the automatic initialization of the lesion distribution is not an easy task to do using only intensity information. Hence, we decided to include local spatial information of the lesion.

Our proposal reduces the user interaction from the empirical choice of parameters to one-click. Thus, the user marks the approximate location of the lesion and the method opens a small window to obtain lesion information and a larger one for the background information. Once the lesion and background information are extracted, the histograms of both regions are calculated and 


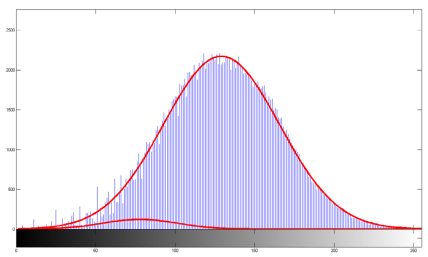

Fig. 2. Gaussian distributions of the US images. The larger Gaussian corresponds to the background and the smaller to the lesion. Both plotted over the image histogram.

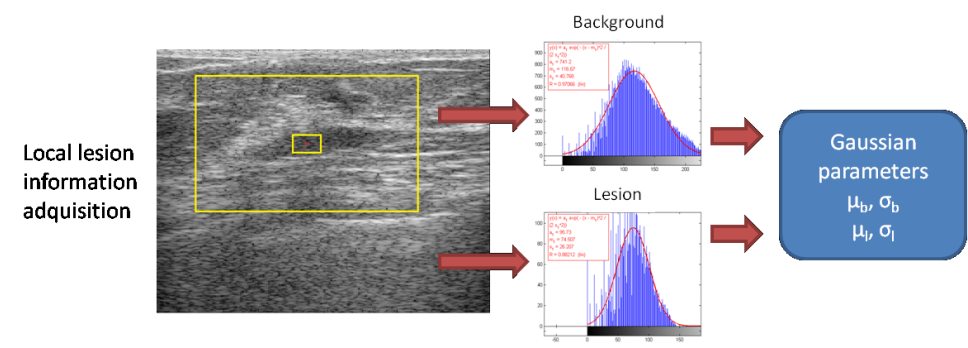

(a)

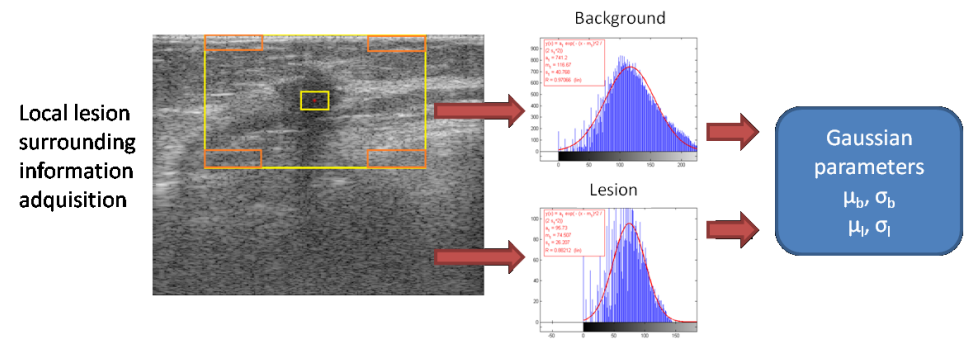

(b)

Fig. 3. (a) Local lesion information acquisition. The central rectangle contains the pixel values for the lesion description and the exterior rectangle contains the background information. (b) Local lesion surrounding information acquisition. The exterior orange rectangles contain the background information.

the best Gaussian distributions are properly fitted to them (see Fig [3(a)). Two different approaches have been extracted from this initialization proposal: Local Lesion Information Global (LLIG) which applies the method to the entire image and Local Lesion Information Partial (LLIP) which crop the image using the background window and applying the segmentation process to this partial image. By cropping the image, the dark regions near image limits are avoided. However, in these proposals the background window could include part of the lesion information so, in order to properly fit the Gaussian distribution to the background histogram, we also propose Local Lesion Surrounding Information (LLSI) which gets histogram information from four small windows surrounding the lesion (see Fig (3) (b). In total, we have evaluated 3 different initialization proposals and compared them to the original method initialization. 
Regarding the final segmentation results, in some cases the method incorrectly classifies parts of the background as a lesion tissue, mostly in the image borders. In contrast with Xiao et al. proposal, a post-processing step is performed, consisting in deleting such regions incorporating information from radiologist knowledge such as the fact that usually the lesion is located centered on the top part of the US image as suggested by Madabhushi and Metaxas in 8 (see Fig. 4(e)).

\section{Experiments and Results}

In order to perform the experiments for this work, a data-set of breast B-mode US images has been collected from the Churchill Hospital of Oxford Radcliffe Hospitals NHS Trust composed by 56 images acquired from 24 different patients, formed by 31 DCIS+IDC (Ductal Carcinoma In Situ + Invasive Ductal Carcinoma), 5 IDC, 6 DCIS, 6 Fibroadenoma, 2 Fibrosis, 1 Cyst, and 5 Mucinous Carcinoma.

\subsection{Ground Truth}

The limited availability of the radiologists made us impossible to have the entire data-set segmented by an expert. Because of that, an extra experiment was performed comparing a subset of 15 segmentations between a radiologist and a non-expert (i.e. biomedical engineer). The experiment obtained a result of 0.7426 for Dice Similarity Coefficient (DSC) and 0.8462 for Area Overlap measures which indicate a high degree of agreement between the non-expert and the radiologist.

\section{$3.2 \quad$ Results}

Fig. 4 shows an example of graphical segmentation results for different images

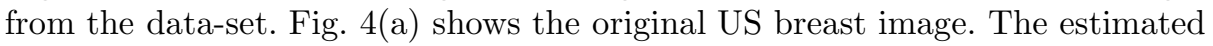
distortion field and the restored image are shown in Fig. 4(b) and (c) respectively. Fig. 4(d) shows the segmentation result and Fig. 4(e) the final result after the post-processing step. Fig. [5 shows the segmentation results using Dice Similarity Coefficient (DSC) and Area Overlap (AO) values for all the images. Analyzing these diagrams for both measures we can see that all the median values of the results for each method are within the confidence interval of the other methods. This means that there is not significative differences between methods and it is not possible to assure that one method obtained better results than another. Note that missegmented images are represented as outliers in the diagram.

Table 1 shows the mean values for sensitivity, specificity, PPV, area overlap and DSC measures. To compare properly all methodologies, all measures were obtained descanting the missegmented images by the original method. Again, there are not significative differences which shows the validity of the proposed initialization. Note that LLIG proposal obtained similar results compared the original implementation while there is not a significant increase of the number of missegmented images. 

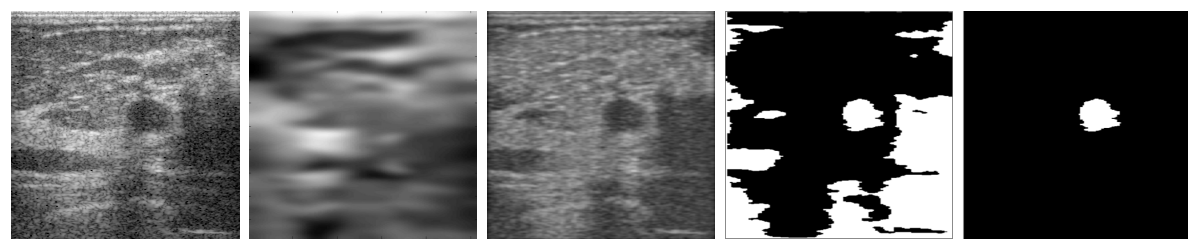

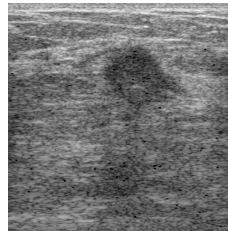

(a)

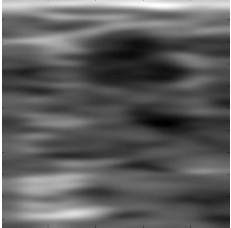

(b)

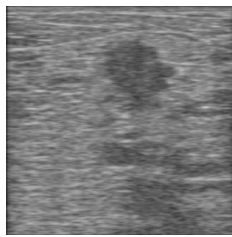

(c)

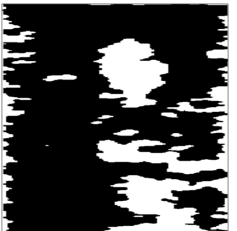

(d)

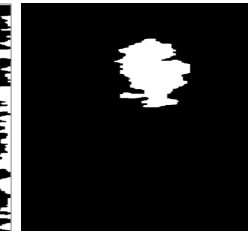

(e)

Fig. 4. Lesion segmentation results. (a) original image, (b) estimated distortion field, (c) corrected image, (d) segmentation result applying LLIG and (e) final segmentation after the segmentation refinement.
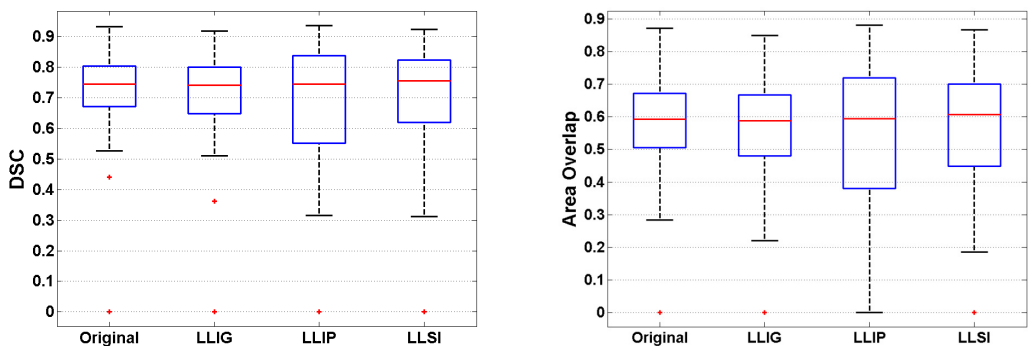

Fig. 5. Box diagrams of DSC and Area Overlap measures for all the methods

Table 1. Comparison between measure means obtained for each method

\begin{tabular}{|l|c|c|c|c|c|c|}
\hline Method & Sensitivity & Specificity & PPV & Area Overlap & DSC & Missegmented Images \\
\hline \hline Original & 0.8978 & 0.7577 & 0.6723 & 0.6110 & 0.7544 & $8 / 56$ \\
LLIG & 0.8701 & 0.7589 & 0.6757 & 0.5996 & 0.7437 & $11 / 56$ \\
LLIP & 0.8035 & 0.6706 & 0.5946 & 0.5538 & 0.6696 & $15 / 56$ \\
LLSI & 0.7889 & 0.7016 & 0.6432 & 0.5848 & 0.6912 & $13 / 56$ \\
\hline
\end{tabular}

\section{Conclusion}

After this study, we have concluded that fully automatic initialization is a hard task using intensity values only. For that reason, we have presented three different approaches where only a one-click interaction is needed. This supposes a high reduction of the user interaction compared to the original approach where a manual selection of the Gaussian parameters was needed. We have compared the obtained results with the original method and we have concluded that all the 
three methods are comparable to the original proposal although LLIG method seems to be more robust and accurate taking into account all the measures used. This lead us to remark that we have improved the original method by means of simplifying the initialization process with no significant loss of correctness.

In order to improve the initialization of the method we propose as a future work to include additional information such as classical features (i.e. SIFT, textons, etc.) or information extracted from the elastography.

\section{References}

1. Besag, J.: On the statistical analysis of dirty pictures. Journal of the Royal Statistical Society B 48, 259-302 (1986)

2. Boukerroui, D., Baskurt, A., Noble, J.A., Basset, O.: Segmentation of ultrasound images: multiresolution $2 \mathrm{~d}$ and $3 \mathrm{~d}$ algorithm based on global and local statistics. Pattern Recognition 24(4-5), 779-790 (2003)

3. Cheng, H., Shi, X., Min, R., Hu, L., Cai, X., Du, H.: Approaches for automated detection and classification of masses in mammograms. Pattern Recognition 39(4), 646-668 (2006)

4. Gil, F., Méndez, I., Sirgo, A., Llort, G., Blanco, I., Cortés-Funes, H.: Perception of breast cancer risk and surveillance behaviours of women with family history of breast cancer: a brief report on a spanish cohort. Psycho-Oncology 12, 821-827 (2003)

5. Horsch, K., Giger, M.L., Venta, L.A., Vyborny, C.J.: Automatic segmentation of breast lesions on ultrasound. Medical Physics 28(8), 1652-1659 (2001)

6. Huang, Y.L., Jiang, Y.R., Chen, D.R., Moon, W.K.: Level set contouring for breast tumor in sonography. J. Digital Imaging 20(3), 238-247 (2007)

7. von Lavante, E., Noble, J.: Segmentation of breast cancer masses in ultrasound using radio-frequency signal derived parameters and strain estimates. In: 5th IEEE International Symposium on Biomedical Imaging: From Nano to Macro, ISBI 2008, pp. 536-539 (May 14-17, 2008)

8. Madabhushi, A., Metaxas, D.: Combining low-, high-level and empirical domain knowledge for automated segmentation of ultrasonic breast lesions. IEEE Transactions on Medical Imaging 22(2), 155-169 (2003)

9. Wells III, W.M., Grimson, W., Kikinis, R., Jolesz, F.: Adaptive segmentation of mri data. IEEE Transactions on Medical Imaging 15(4), 429-442 (1996)

10. Xiao, G., Brady, M., Noble, J., Zhang, Y.: Segmentation of ultrasound b-mode images with intensity inhomogeneity correction. IEEE Transactions on Medical Imaging 21(1), 48-57 (2002)

11. Yeh, C.K., Chen, Y.S., Fan, W.C., Liao, Y.Y.: A disk expansion segmentation method for ultrasonic breast lesions. Pattern Recognition 42(5), 596-606 (2009)

12. Zouqi, M., Samarabandu, J.: 2d ultrasound image segmentation using graph cuts and local image features. In: IEEE Symposium on Computational Intelligence for Image Processing, CIIP 2009, pp. 33-40 (March 2009) 